\title{
Odonates from Bodoquena Plateau: checklist and information about endangered species
}

\author{
Ricardo Koroiva $^{1 *}$, Marciel Elio Rodrigues ${ }^{2}$, Francisco Valente-Neto ${ }^{I}$ \& Fábio de Oliveira Roque ${ }^{I}$ \\ ${ }^{1}$ Universidade Federal do Mato Grosso do Sul, Instituto de Biociências, Cidade Universitária, \\ 79070-900, Campo Grande, MS, Brazil \\ ${ }^{2}$ Universidade Estadual de Santa Cruz, Departamento de Ciências Biológicas, Rod. Jorge Amado, \\ km 16, 45662-900, Ilhéus, BA, Brazil \\ *Corresponding author: Ricardo Koroiva, e-mail: ricardo.koroiva@gmail.com
}

KOROIVA, R., RODRIGUES, M.E., VALENTE-NETO, F., ROQUE, F.O. Odonates from Bodoquena Plateau: checklist and information about endangered species. Biota Neotropica. 17(3): e20160310. http://dx.doi.org/10.1590/16760611-BN-2016-0310

\begin{abstract}
Here we provide an updated checklist of the odonates from Bodoquena Plateau, Mato Grosso do Sul state, Brazil. We registered 111 species from the region. The families with the highest number of species were Libellulidae (50 species), Coenagrionidae (43 species) and Gomphidae (12 species). 35 species are registered in the IUCN Red List species, four being Data Deficient, 29 of Least Concern and two species being in the threatened category. Phyllogomphoides suspectus Belle, 1994 (Odonata: Gomphidae) was registered for the first time in the state.
\end{abstract}

Keywords: Dragonfly, Damselfly, inventory, Cerrado, Brazil

\section{Libélulas da Serra da Bodoquena: lista de espécies e informações sobre espécies ameaçadas}

\begin{abstract}
Resumo: Nós apresentamos um inventário atualizado das espécies de libélulas ocorrentes na Serra da Bodoquena, Estado de Mato Grosso do Sul, Brasil. Nós registramos 111 espécies para a região. As famílias com o maior número de espécies foram Libellulidae (50 espécies), Coenagrionidae (43 espécies) e Gomphidae (12 espécies). 35 espécies são registradas na Lista Vermelha de espécies da IUCN e duas estão em categorias de ameaçadas. Phyllogomphoides suspectus Belle, 1994 (Odonata: Gomphidae) foi registrado pela primeira vez no estado.
\end{abstract}

Palavras-chave: Libélulas, donzelinhas, Inventário, Cerrado, Brasil

\section{Introduction}

Insects are arthropod group with largely unknown distribution patterns (Diniz-Filho et al. 2010), due to their high species richness, difficulties related to sampling and lack of taxonomic experts. Applying all of these conditions, odonates (Insecta: Odonata) constitutes an important group that play a major role as predators, with several insects making up a large portion of their diets. This group is also a good indicators of water quality, with their nymphs being an important link in food chains for fish and other aquatic vertebrates and serve as an inspired subject for artists, naturalists and collectors (Abbott 2015).

Even with important taxonomists in the recent Brazilian history, odonates are still poorly described and its distribution is deficiently known in the Neotropical region (Kalkman et al. 2008). Local inventories are recorded to several parts of Brazil like São Paulo (Costa et al. 2000), Espirito Santo (Costa \& Oldrini 2005), Rio de Janeiro (Anjos-Santos \& Costa 2006), Goiás and Distrito Federal (Nóbrega \& De Marco 2011), Minas Gerais (Souza et al. 2013), and Rio Grande do Sul (Renner et al. 2016). In Mato Grosso do Sul, state originally covered by Cerrado, Pantanal, Atlantic Forest and Chaco vegetation, 198 odonatan species were registered (Rodrigues \& Roque 2017). Despite this number, information gaps are recognized from many areas in this state (Vianna \& De Marco 2012, Rodrigues \& Roque 2017).
Bodoquena Plateau is a threatened area in the Mato Grosso do Sul state because of the broad conversion of natural areas to pastures and croplands (Roque et al. 2016). In the last five years, several research projects about biodiversity and the ecology of aquatic insects have been developed in Bodoquena Plateau, (e.g. LTER Planalto da Bodoquena, Dragonflies of Serra da Bodoquena Project and Observagua Project). These projects have promoted a great opportunity for extending sampling effort of aquatic insects, especially for odonates (e.g. Koroiva et al. 2016, Rodrigues et al. 2016a, Rodrigues et al. 2016b, Valente-Neto et al. 2016). Until 2011, about 68 Odonata species were registered to Bodoquena Plateau; a number that was considered underestimated because of the broad unsampled area (Dalzochio et al. 2011b). Therefore, even with the publications of checklists (Dalzochio et al. 2011a, Dalzochio et al. 2011b, Rodrigues \& Roque 2017), the amount of information generated by these new sampling efforts and visits to museums demand an update.

Here, we provide an updated checklist of the odonates from Bodoquena Plateau, including species records from recent sampling efforts, from the literature, and from specimens deposited in the Reference Collection of the Federal University of Mato Grosso do Sul (CEUFMS). We also provide information about endangered species from the IUCN Red List (IUCN 2016), which is fundamental for updating the status of Brazilian species, as recently emphasized in the $1^{\text {st }}$ Meeting of the Latin American Odonatological Society - SOL. 


\section{Material and Methods}

\section{Sources of data}

We used different sources of information to produce the checklist, including samplings (from 2011 to 2016), previous available records on literature and information collected in zoological reference collections of the Mato Grosso do Sul state. For each source, we searched for names and geographic coordinates of the locations. For records that did not have latitude and longitude values, geographic coordinates of the centroid of the place where the site is located.

\section{Sampling in study region}

All the sampling sites were located within Bodoquena Plateau region ( $21^{\circ} 08^{\prime} 02^{\prime \prime}$ to $20^{\circ} 38^{\prime} 26^{\prime \prime}$ S and $56^{\circ} 48^{\prime} 31^{\prime \prime}$ to $\left.56^{\circ} 44^{\prime} 28^{\prime \prime} \mathrm{W}\right)$. The Bodoquena Plateau is located in the mid-southern portion of Mato Grosso do Sul State, including the cities of Bodoquena, Bonito and Jardim. The region is characterized by a mountain chain, with altitudes varying between 450 and $800 \mathrm{~m}$. The climate is temperate humid with hot summers and two well-defined seasons. The average annual temperature varies between 20 and $22^{\circ} \mathrm{C}$ and annual rainfall varies between 1300 and $1700 \mathrm{~mm}$. The hydrography of the region is characterized by limestone, rivers with high alkalinity and low turbidity. Its tributaries are part of the Paraguay River watershed (PCBAP 1997).

Bodoquena Plateau has both areas of the Cerrado (Brazilian savanna) and Atlantic Forest biomes. This region is an important ecological corridor for biodiversity and one of the most important areas of Ecotourism in Brazil (Klein et al. 2011). Considered a priority area for conservation by the Brazilian Ministry of Environment (MMA) (Brasil 2016), the region is currently threatened by inadequate exploitation of its resources and the progressive agricultural expansion.
The dataset used in this checklist included 85 lotic environments (Figure 1) sampled during the period between 2011 and 2016. These samples were concentrated on adults from Odonata and were performed during Ph.D studies (Valente-Neto 2015, Rodrigues 2016), Long term ecological research in the Bodoquena Plateau (LTER-Serra da Bodoquena), Dragonflies of Serra da Bodoquena Project and Observagua Project. The sampling method used handhold insect nets, in sunny days, during the peak time of Odonata activities (between 09:00 h to 16:00 h). The collection authorization process was issued by IBAMA, through SISBio system under the number 41313-3 and 54386-1. All specimens collected were deposited in the Reference Collection of the Universidade Federal de Mato Grosso do Sul (CEUFMS), afterwards determined in laboratory according to Garrison et al. (2006, 2010), Heckman (2006, 2008) and Lencioni $(2005,2006)$. For the systematics classification, we followed Dijkstra et al. (2014) and for scientific names and followed the synonymic list of Garrisson \& von Ellenrieder (2016). In the laboratory, dragonflies were analyzed under Zeiss Discovery V20 stereoscope and recorded with an AxioCam Icc 5 Microscope Camera. For capturing and editing images, we used the software Zen 2-2011.

\section{Data analysis}

We estimated the sampling accuracy of sampling sites with incidence-based non-parametric estimators: Chao 2, Jacknife 1 and Jacknife 2. Non-parametric methods have better performance compared to others estimators for aquatic insects (Melo \& Froelich 2001). These analyses were performed with EstimateS version 9.1.0 software (Colwell 2013). We set 1000 repetitions to calculated mean values with confidence intervals of $95 \%$.

\section{Literature and reference collections information}

Aiming to improve our list of species, we used online available databases to find registers of Odonata species from Bodoquena Plateau, specifically Web of Knowledge (which includes the main journals of world), Scielo (which include the main journals of South America) and Google Scholar.

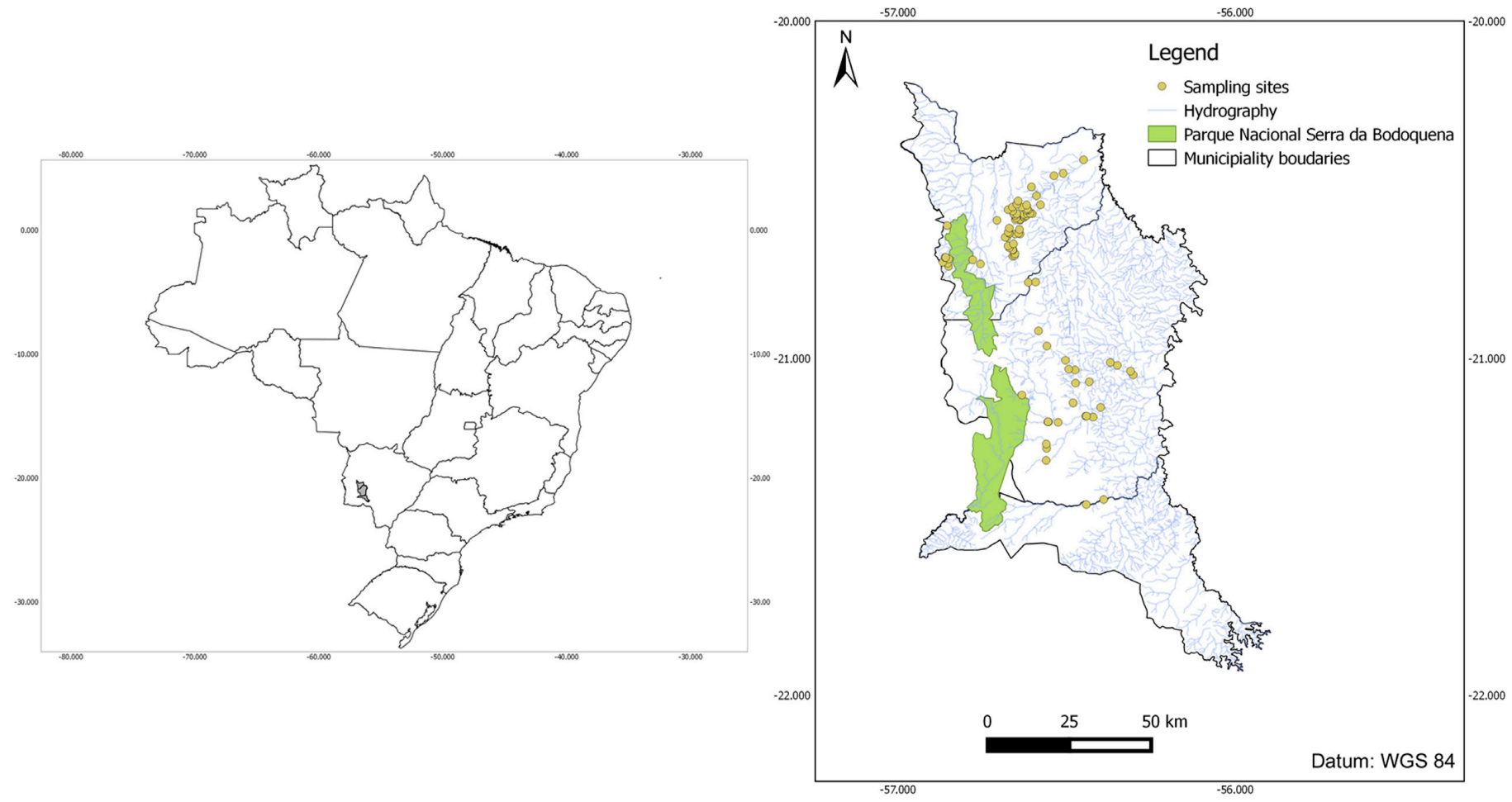

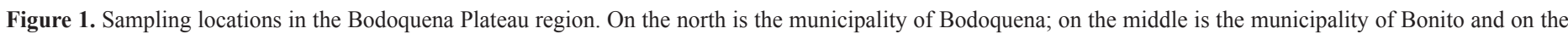
south is the municipality of Jardim. 
We did the search on general keywords like "Odonata", "dragonfly" or "libélula" ("Dragonfly" in Portuguese) in combination with "Mato Grosso do Sul", "Bodoquena Plateau" or "Serra da Bodoquena" ("Bodoquena Plateau" in Portuguese). Souza (2003), Dalzochio et al. (2011a) and Dalzochio et al. (2011b) provided additional information about registers of Odonata species ("Reference" in Table 1).
In addition, we analyzed the deposited specimens from two important Zoological collections of Mato Grosso do Sul state: Reference Collection Universidade Federal de Mato Grosso do Sul (CEUFMS) and Biodiversity Museum of Universidade Federal da Grande Dourados (UFGD). CEUFMS provided additional register besides that found in the project samples or in literature ("CEUFMS" in Table 1).

Table 1. Inventory list of Odonata species from Bodoquena Plateau with information about endangered species (IUCN). CR $=\mathrm{Critically} \mathrm{Endangered,} \mathrm{VU}=\mathrm{Vulnerable}$, $\mathrm{LC}=$ Least Concern, DD = Data Deficient; *, first record to Bodoquena Plateau.

\begin{tabular}{|c|c|c|c|c|}
\hline Suborder & Family & Species & Source & $\begin{array}{c}\text { IUCN Red list } \\
\text { status }\end{array}$ \\
\hline \multirow[t]{42}{*}{ Anisoptera } & \multirow[t]{2}{*}{ Aeshnidae } & Castoraeschna sp Calvert, 1952* & This study (BOMS 0052/UFMS BOMS 0053/UFMS) & - \\
\hline & & Staurophlebia reticulata Burmeister, $1839^{*}$ & This study (CANI 414/UFMS) & - \\
\hline & \multirow[t]{12}{*}{ Gomphidae } & Agriogomphus ericae Belle, 1966 & Reference (Dalzochio et al. 2011b) & - \\
\hline & & Archaeogomphus sp Williamson, 1919 & Reference (Dalzochio et al. 2011b) & - \\
\hline & & Cyanogomphus sp Selys, 1873 & Reference (Dalzochio et al. 2011b) & - \\
\hline & & Gomphoides infumata Rambur, 1842 & Reference (Dalzochio et al. 2011a) & - \\
\hline & & Phyllogomphoides suspectus Belle, 1994 * & This study (6-R15-04/UFMS & DD \\
\hline & & Phyllocycla sp Calvert, 1948 & This study (CANI336/UFMS) & - \\
\hline & & Progomphus amazonicus Belle, 1973 & Reference (Dalzochio et al. 2011a) & DD \\
\hline & & Progomphus intricatus Hagen in Selys, 1858 & Reference (Dalzochio et al. 2011b) & $\mathrm{LC}$ \\
\hline & & Progomphus montanus Belle, 1973 & Reference (Dalzochio et al. 2011a) & $\mathrm{LC}$ \\
\hline & & Progomphus sp1 Selys, 1854 & This study (CANI 411/UFMS) & - \\
\hline & & Progomphus sp2 Selys, 1854 & This study (CANI 334/UFMS) & - \\
\hline & & Remartinia sp Navás, $1911^{*}$ & This study (CANI 017/UFMS) & - \\
\hline & \multirow{28}{*}{ Libellulidae } & Brechmorhoga sp Kirby, 1894 & Reference (Dalzochio et al. 2011b) & - \\
\hline & & Dythemis sterilis Hagen, 1861 & This study (6-R15-21/UFMS) & - \\
\hline & & Elasmothemis cannacrioides Calvert, 1906 * & $\begin{array}{l}\text { This study (6-R15-28/UFMS, 6-R15-14/UFMS, } \\
\text { 6-R15-8/UFMS) }\end{array}$ & - \\
\hline & & Elasmothemis williamsoni Ris, 1919 & Reference (Dalzochio et al. 2011a) & - \\
\hline & & Elga newtonsantosi Machado, 1992 * & This study (CANI 833/UFMS, CANI 413/UFMS) & $\mathrm{CR}$ \\
\hline & & Erythemis vesiculosa Fabricius, 1775 & This study (1-R19-3/UFMS) & $\mathrm{LC}$ \\
\hline & & Erythemis mithroides Brauer in Therese, 1900 * & CEUFMS (CEUFMS 6048) & $\mathrm{LC}$ \\
\hline & & Erythemis peruviana Rambur, $1842 *$ & $\begin{array}{l}\text { CEUFMS (CEUFMS 6044, CEUFMS 6046, } \\
\text { CEUFMS 6047) }\end{array}$ & - \\
\hline & & Erythemis plebeja Burmeister, 1839 & CEUFMS (CEUFMS 6043) & $\mathrm{LC}$ \\
\hline & & Erythrodiplax basalis Kirby, $1897^{*}$ & $\begin{array}{l}\text { This study (CANI 011/UFMS, CANI 015/UFMS, } \\
\text { CANI 09/UFMS, CANI 222/UFMS) }\end{array}$ & - \\
\hline & & Erythrodiplax branconensis Sjöstedt, 1929 * & This study (CANI 217/UFMS) & - \\
\hline & & Erythrodiplax famula Erichson in Schomburgk, 1848 & Reference (Dalzochio et al. 2011a) & - \\
\hline & & Erythrodiplax fusca Rambur, 1842 & $\begin{array}{l}\text { This study (3-R14-11/UFMS, 3-R14-10/UFMS, } \\
\text { 3-R14-24/UFMS, 6-R15-3/UFMS) }\end{array}$ & - \\
\hline & & Erythrodiplax kimminsi Borror, $1942^{*}$ & This study (CANI 088/UFMS) & - \\
\hline & & Erythrodiplax lativittata Borror, 1942 * & $\begin{array}{l}\text { This study (CANI 405/UFMS, CANI 499/UFMS, } \\
\text { CANI 505/UFMS, CANI 502/UFMS) }\end{array}$ & - \\
\hline & & Erythrodiplax ochracea Burmeister, 1839 & $\begin{array}{l}\text { This study (8-R22-1/UFMS, 8-R22-4/UFMS, 8-R22- } \\
\text { 5/UFMS) }\end{array}$ & $\mathrm{LC}$ \\
\hline & & Erythrodiplax paraguayensis Förster, 1905 & This study (BNBTO-0005/UFMS) & $\mathrm{LC}$ \\
\hline & & Erythrodiplax umbrata Linnaeus, 1758 & $\begin{array}{l}\text { This study (7-R21-1/UFMS, 7-R21-2/UFMS, 7-R21- } \\
\text { 4) }\end{array}$ & - \\
\hline & & Macrothemis flavescens Kirby, 1897 * & This study (CANS 480/UFMS) & $\mathrm{LC}$ \\
\hline & & Macrothemis hemichlora Burmeister, 1839 & This study (BOMS-0328/UFMS) & $\mathrm{LC}$ \\
\hline & & Macrothemis heteronycha Calvert, 1909 & Reference (Dalzochio et al. 2011b) & $\mathrm{LC}$ \\
\hline & & Macrothemis imitans imitans Karsch, 1890 & Reference (Dalzochio et al. 2011a) & - \\
\hline & & Macrothemis sp Hagen, $1868^{*}$ & This study (BOMS0379/UFMS) & - \\
\hline & & Miathyria marcella Selys in Sagra, 1857 & This study (2-R16-3/UFMS, 7-R21-6/UFMS) & $\mathrm{LC}$ \\
\hline & & Micrathyria catenata Calvert, 1909 & Reference (Souza 2003) & $\mathrm{LC}$ \\
\hline & & Micrathyria hesperis Ris, 1911 & $\begin{array}{l}\text { CEUFMS (CEUFMS 6019, CEUFMS 6020, } \\
\text { CEUFMS 6021) }\end{array}$ & - \\
\hline & & Micrathyria laevigata Calvert, 1909 & This study (CANI 019/UFMS) & - \\
\hline & & Micrathyria longifasciata Calvert, 1909 & Reference (Dalzochio et al. 2011b) & $\mathrm{LC}$ \\
\hline
\end{tabular}


Table 1. Continued..

\begin{tabular}{|c|c|c|c|c|}
\hline Suborder & Family & Species & Source & $\begin{array}{l}\text { IUCN Red list } \\
\text { status }\end{array}$ \\
\hline & & Micrathyria ocellata Martin, 1897 & $\begin{array}{l}\text { CEUFMS (CEUFMS 5998, CEUFMS 5999, CEUFM } \\
6008)\end{array}$ & - \\
\hline & & Micrathyria pseudeximia Westfall, 1992 & Reference (Souza 2003) & - \\
\hline & & $\begin{array}{l}\text { Micrathyria pseudhypodidyma Costa, Lourenço \& Viera, } \\
2002^{*}\end{array}$ & This study (CANI 433/UFMS, CANI 432/UFMS) & VU \\
\hline & & Micrathyria stawiarskii Santos, $1953^{*}$ & This study (BOMS0076/UFMS) & $\mathrm{LC}$ \\
\hline & & Micrathyria tibialis Kirby, 1897 & Reference (Souza 2003) & $\mathrm{LC}$ \\
\hline & & Micrathyria ungulata Förster, $1907^{*}$ & This study (CANI 013/UFMS) & - \\
\hline & & Oligoclada sp. Karsch, 1890 & Reference (Souza 2003) & - \\
\hline & & Orthemis cultriformis Calvert, $1899^{*}$ & This study (BNBTO202-12/UFMS) & - \\
\hline & & Orthemis discolor Burmeister, 1839 & This study (1-R19-7/UFMS) & - \\
\hline & & Orthemis ferruginea Fabricius, $1775^{*}$ & $\begin{array}{l}\text { CEUFMS (CEUFMS 5886, CEUFMS 5888, } \\
\text { CEUFMS 5889) }\end{array}$ & $\mathrm{LC}$ \\
\hline & & Orthemis schmidti Buchholz, 1950 & CEUFMS (CEUFMS 59891) & - \\
\hline & & Pantala flavescens Fabricius, 1798 & This study (BOMS0088/UFMS) & $\mathrm{LC}$ \\
\hline & & Pantala hymenae Say, $1839^{*}$ & This study (CANI 041/UFMS) & - \\
\hline & & Perithemis electra Ris, $1930^{*}$ & $\begin{array}{l}\text { This study (CANI 588/UFMS, CANI 514/UFMS, } \\
\text { CANI 517/UFMS, CANI 501/UFMS) }\end{array}$ & $\mathrm{LC}$ \\
\hline & & Perithemis icteroptera Selys in Sagra, $1857^{*}$ & This study (13-R31-2/UFMS) & $\mathrm{LC}$ \\
\hline & & Perithemis lais Perty, 1834 & This study (CANI 301/UFMS, CANI 459/UFMS) & $\mathrm{LC}$ \\
\hline & & Perithemis mooma Kirby, 1889 & This study (CANI 460/UFMS) & - \\
\hline & & Perithemis thais Kirby, 1889 & $\begin{array}{l}\text { This study (CANI 481/UFMS, CANI 468/UFMS, } \\
\text { CANI 464/UFMS, CANI 463/UFMS) }\end{array}$ & - \\
\hline & & Tauriphila australis Hagen, 1867 & Reference (Souza 2003) & - \\
\hline & & Tramea cophysa Hagen, $1867^{*}$ & $\begin{array}{l}\text { CEUFMS (CEUFMS 6082, CEUFMS 6093, } \\
\text { CEUFMS 6084) }\end{array}$ & - \\
\hline & & Tramea minuta De Marmels \& Rácenis, 1982 & Reference (Dalzochio et al. 2011a) & - \\
\hline & & Tramea rustica De Marmels \& Rácenis, 1982 * & $\begin{array}{l}\text { CEUFMS (CEUFMS 6074, CEUFMS } 6075 \text {, } \\
\text { CEUFMS 6076) }\end{array}$ & $\mathrm{LC}$ \\
\hline \multirow[t]{22}{*}{ Zygoptera } & Calopterygidae & Hetaerina mortua Hagen in Selys, $1853^{*}$ & This study (CZYG 693/UFMS) & - \\
\hline & & Hetaerina rosea Selys, 1853 & $\begin{array}{l}\text { This study (6-R15-23/UFMS, 02-R16-01/UFMS, } \\
\text { 4-R17-1/UFMS, 4-R17-2/UFMS) }\end{array}$ & - \\
\hline & Coenagrionidae & Acanthagrion aepiolum Tennessen, 2004 & $\begin{array}{l}\text { This study (12-R25-18/UFMS, 9-R27-5/UFMS, 10- } \\
\text { R28-8/UFMS, 13-R30-16/UFMS) }\end{array}$ & - \\
\hline & & Acanthagrion apicale Selys, 1876 & Reference (Dalzochio et al. 2011a) & - \\
\hline & & Acanthagrion ascendens Calvert, 1909 & Reference (Dalzochio et al. 2011a) & - \\
\hline & & Acanthagrion chararum Calvert, 1909 & Reference (Dalzochio et al. 2011b) & - \\
\hline & & Acanthagrion cuyabae Calvert, 1909 & $\begin{array}{l}\text { This study (10-R28-2/UFMS, 10-R28-6/UFMS, 10- } \\
\text { R28-7/UFMS) }\end{array}$ & $\mathrm{LC}$ \\
\hline & & Acanthagrion gracile Rambur, 1842 & $\begin{array}{l}\text { This study (8-R22-2/UFMS, 12-R25-20/UFMS, 12- } \\
\text { R25-17/UFMS) }\end{array}$ & - \\
\hline & & Acanthagrion temporale De Marmels, 1985 & Reference (Souza 2003) & - \\
\hline & & Acanthagrion truncatum Selys, 1876 & Reference (Souza 2003) & $\mathrm{LC}$ \\
\hline & & Aeolagrion dorsale Burmeister, $1839^{*}$ & This study (CZYG 1011/UFMS, CZYG 1551/UFMS) & - \\
\hline & & Argentagrion ambiguum Ris, $1904^{*}$ & CEUFMS (CZYGO 5001/UFMS) & - \\
\hline & & Argia chapadae Calvert, $1909^{*}$ & $\begin{array}{l}\text { This study (5-R13-1/UFMS, 3-R14-16/UFMS, 6-R15- } \\
\text { 1/UFMS, 1-R19-10/UFMS) }\end{array}$ & - \\
\hline & & Argia croceipennis Selys, 1865 & $\begin{array}{l}\text { This study (CZYG 1504/UFMS, CZYG 1837/UFMS, } \\
\text { CZYG 1328/UFMS, CZYG 1329/UFMS) }\end{array}$ & $\mathrm{LC}$ \\
\hline & & Argia cupraurea Calvert, 1902 & Reference (Souza 2003) & - \\
\hline & & Argia hasemani Calvert, $1909^{*}$ & $\begin{array}{l}\text { This study (CZYG 1779/UFMS, CZYG 1791/UFMS, } \\
\text { CZYG 501/UFMS) }\end{array}$ & - \\
\hline & & Argia indocilis Navás, 1934 & This study (CZYG 873/UFMS) & - \\
\hline & & Argia lilacina Selys, $1865^{*}$ & This study (CZYG 412/UFMS) & - \\
\hline & & Argia modesta Selys, $1865^{*}$ & This study (BNBTO-0288/UFMS) & - \\
\hline & & Argia mollis Hagen in Selys, $1865^{*}$ & This study (3-R14-17/UFMS) & - \\
\hline & & Argia reclusa Selys, $1865^{*}$ & $\begin{array}{l}\text { This study (6-R15-16/UFMS, 1-R19-11/UFMS, } \\
\text { 9-R27-1/UFMS, 13-R30-2/UFMS) }\end{array}$ & - \\
\hline & & Argia smithiana Calvert, $1909^{*}$ & This study (BNBTO-0093) & - \\
\hline
\end{tabular}


Table 1. Continued...

\begin{tabular}{|c|c|c|c|c|}
\hline Suborder & Family & Species & Source & $\begin{array}{l}\text { IUCN Red list } \\
\text { status }\end{array}$ \\
\hline & & Argia subapicalis Calvert, $1909^{*}$ & This study (CZYG 509/UFMS) & $\mathrm{DD}$ \\
\hline & & Argia tamoyo Calvert, 1909 & This study (BNBTO-0289/UFMS) & - \\
\hline & & Enallagma novaehispaniae Calvert, 1907 & $\begin{array}{l}\text { This study (CZYG 742/UFMS, CZYG 1128/UFMS, } \\
\text { CZYG 1137/UFMS) }\end{array}$ & - \\
\hline & & Epipleoneura metallica Rácenis, $1955^{*}$ & This study (BNBTO244-12/UFMS) & $\mathrm{LC}$ \\
\hline & & Epipleoneura venezuelensis Rácenis, 1955 & $\begin{array}{l}\text { This study (CZYG 958/UFMS, CZYG 1112/UFMS, } \\
\text { CZYG 1116/UFMS) }\end{array}$ & - \\
\hline & & Forcepsioneura sp. Lencioni, 1999 & Reference (Souza 2003) & - \\
\hline & & Homeoura chelifera Selys, 1876 & Reference (Dalzochio et al. 2011a) & - \\
\hline & & Homeoura nepos Selys, $1876^{*}$ & This study (CZYG 433/UFMS, CZYG 437/UFMS) & - \\
\hline & & Ischnura capreolus Hagen, 1861 & Reference (Souza 2003) & - \\
\hline & & Ischnura fluviatilis Selys, 1876 & Reference (Souza 2003) & $\mathrm{LC}$ \\
\hline & & Metaleptobasis lillianae Daigle, 2004 * & This study (CZYG 1358/UFMS, CZYG 1359/UFMS) & DD \\
\hline & & Neoneura bilinearis Selys, 1860 * & $\begin{array}{l}\text { This study (CZYG 2091/UFMS, CZYG 2101/UFMS, } \\
\text { CZYG 2099/UFMS) }\end{array}$ & $\mathrm{LC}$ \\
\hline & & Neoneura ethela Williamson, 1917 & $\begin{array}{l}\text { This study (CZYG 1815/UFMS, CZYG 1828/UFMS, } \\
\text { CZYG 1397/UFMS) }\end{array}$ & - \\
\hline & & Neoneura rubriventris Selys, 1860 & $\begin{array}{l}\text { This study (CZYG 8908/UFMS, CZYG 8907/UFMS, } \\
\text { CZYG 8906/UFMS }\end{array}$ & - \\
\hline & & Neoneura sylvatica Hagen in Selys, 1886 & $\begin{array}{l}\text { This study (05-R13-09/UFMS, 06-R15-31/UFMS, } \\
\text { 13-R30-22/UFMS) }\end{array}$ & - \\
\hline & & Oxyagrion chapadense Costa, 1978 & Reference (Dalzochio et al. 2011b) & - \\
\hline & & $\begin{array}{l}\text { Oxyagrion sulmatogrossense Costa, Souza \& Santos, } \\
2000^{*}\end{array}$ & This study (9-R27-3/UFMS) & - \\
\hline & & Oxyagrion terminale Selys, $1876^{*}$ & This study (CZYG 189/UFMS) & - \\
\hline & & Peristicta aeneoviridis Calvert, 1909 & $\begin{array}{l}\text { This study (CEUFMS 8925, CEUFMS } 8926 \text {, } \\
\text { CEUFMS 8924) }\end{array}$ & - \\
\hline & & Peristicta muzoni Pessacq \& Costa, 2007 & Reference (Dalzochio et al. 2011b) & - \\
\hline & & Telebasis sanguinalis Calvert, 1909 & Reference (Souza 2003) & - \\
\hline & & Telebasis willinki Fraser, 1948 & Reference (Dalzochio et al. 2011a) & $\mathrm{LC}$ \\
\hline \multirow{3}{*}{\multicolumn{2}{|c|}{ Lestidae }} & Tigriagrion aurantinigrum Calvert, 1909 * & This study (13-R30-24/UFMS) & - \\
\hline & & Lestes forficula Rambur, 1842 & $\begin{array}{l}\text { This study (CEUFMS 8903, CEUFMS } 8902 \text {, } \\
\text { CEUFMS 8901) }\end{array}$ & - \\
\hline & & Lestes curvatus Belle, 1997 & Reference (Souza 2003) & $\mathrm{LC}$ \\
\hline
\end{tabular}

\section{Results}

\section{Species list}

Sampling projects data recorded 67 species, belonging to 42 genera in six families, totaling 1902 collected specimens. The estimators showed that this richness corresponds to $79.99,76.35$ and $69.99 \%$ of the medium estimated richness by, respectively, Chao 2 (Sest $=83.76$, standard deviation. $= \pm 9.59)$, Jackknife $1($ Sest $=87.75$, standard deviation $= \pm 4.85)$ and Jackknife 2 (Sest =95.72). The accumulation curves obtained are non-asymptotic (Figure 2), thus indicating that total species richness would probably increase with additional sampling effort.

The Odonata list records from the Bodoquena Plateau are presented in Table 1. We added another fourty-four species that were registered in the literature ( 34 species) or deposited in the Reference Collection of the Federal University of Mato Grosso do Sul (10 species). Thus, we have considered the presence of 111 Odonata species. We recorded for the first time the presence of 42 species from Bodoquena Plateau ( 24 anisopterans and 18 zygopterans). In this inventory list, 35 species are registered in IUCN red List (IUCN 2016), with four being in Data Deficient (DD), 29 in Least Concern (LC) and two species are in threatened categories, Elga newtonsantosi Machado, 1992 like Critically Endangered (CR)

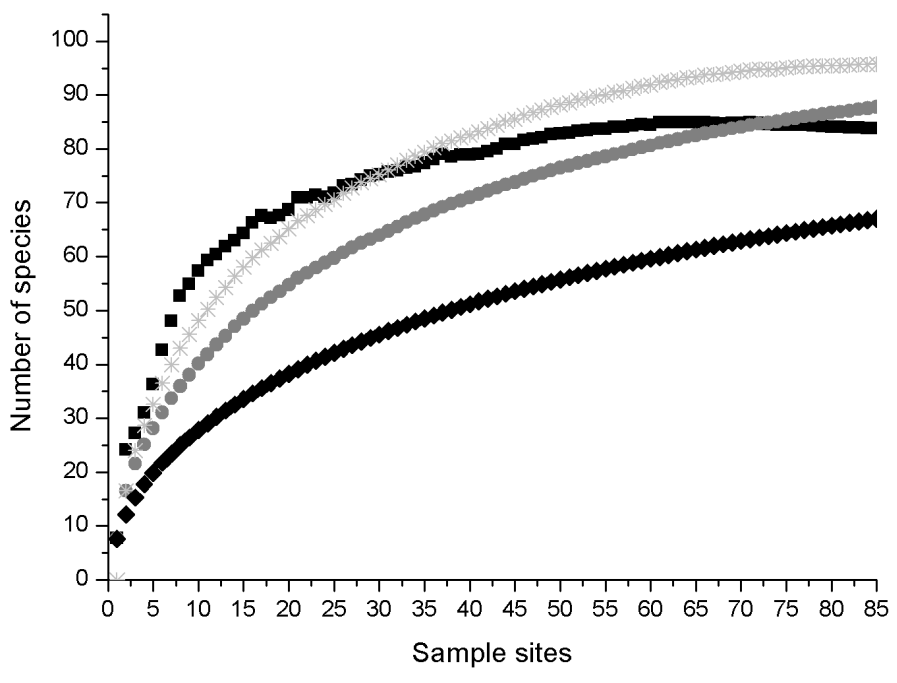

Figure 2. Accumulation curves for observed and estimated number of species collected at 85 sample points. The estimates were calculated with the estimator Chao 2, Jackknife 1 and Jackknife 2. Black diamonds = number of species observed (est); Black squares $=$ Chao 2 richness estimator; Grey circles $=$ Jackknife 1 richness estimator; Grey stars = Jackknife 2 richness estimator . All curves are plotted form the means of 1000 randomizations of sample accumulation order. 
and Micrathyria pseudhypodidyma Costa, Lourenço \& Viera, 2002 like Vulnerable (VU).

The families with the higher number of species were Libellulidae (45.05\%), Coenagrionidae (38.74\%) and Gomphidae (10.81\%) (Table 2). The most speciose genus were Argia, Micrathyria and Erythrodiplax, represented by twelve, ten and nine species, respectively. The endangered species classified in the IUCN Red List, Elga newtonsantosi and Micrathyria pseudhypodidyma, were registered only in two and one sampled sites, respectively.

\section{New record}

We found that Phyllogomphoides suspectus Belle, 1994 represents a new records of odonate species for Mato Grosso do Sul state, compared with the list published by Rodrigues \& Roque (2017). The genus Phyllogomphoides Belle, 1970, is composed of 45 species in the Neotropical Region, 29 of these in South America (Belle 1994). Phyllogomphoides suspectus Belle, 1994 has a distribution recorded by Belle (1994) and von Ellenrieder (2009a) and is considered exclusive to Brazil. The species distribution ranges from Rondonia to the Mato Grosso with specimens found in Rio Ouro Preto and Sinop, respectively (Belle 1994), around $800 \mathrm{~km}$ northwards of our sampling site.

We collected one male adult of Phyllogomphoides in the rural area of Bonito, Mato Grosso do Sul state, Brazil. This specimen was identified as P. suspectus (Figure 3a), according to the characteristics indicated by Belle (1994) and Heckman (2008), like spines along ventral tergal margins of abdominal segment 7 small and largely black (Figure $3 \mathrm{~b}$ ), the cerci, in

Table 2. Number of Odonata species collected per family in the Bodoquena Plateau.

\begin{tabular}{lcc}
\hline SUBORDER FAMILY & Number & $\%$ \\
\hline ANISOPTERA & 2 & 1.80 \\
Aeshnidae & 12 & 10.81 \\
Gomphidae & 50 & 45.05 \\
Libellulidae & & \\
ZYGOPTERA & 2 & 1.80 \\
Calopterygidae & 43 & 38.74 \\
Coenagrionidae & 2 & 1.80 \\
Lestidae & 111 & 100 \\
TOTAL & &
\end{tabular}

dorsal view, with forcipate shape (Figure 3c) and with a hatchet-shaped inferior protuberance near the base (Figure $3 \mathrm{~d}$ ) and anterior genital hamules without a posterior black hook (Figure 3e).

\section{Discussion}

Inventories promote skeletal support in many fields, including biogeography, population and community ecology. Such data establish a baseline of biodiversity and ecological information, which allow their application in conservation strategies. The list of species shown here represents $55.77 \%$ of all species recorded for Mato Grosso do Sul (199 species) (Rodrigues \& Roque 2017, Rodrigues et al. 2016a), and is a significant advance in comparison with previous accounts (Souza 2013, Dalzochio et al. 2011a, Dalzochio et al. 2011b), increasing by 42 odonate species list of Bodoquena Plateau region.

Considering that insect inventories, especially those of very speciesrich taxa in tropical region, are often incomplete (Gotelli \& Colwell 2001), our results show that accumulation curves have not tended to stabilize. The reference source emphasize this result, because there are 44 species registered by Souza (2003) and Dalzochio et al. (2011a, 2011b) and not sampled by the projects. Other reasons for the high number of non-sampled species are the large territory of the Bodoquena Plateau and many of these species had been collected in the southern part, region not sampled by the projects. Furthermore, the projects sample sites, like the other previous studies (Souza 2003, Dalzochio et al. 2011a, Dalzochio et al. 2011b), focused on lotic system rather lentic ones, such as lakes and reservoirs. Thus, future inventories in lentic environments and with other sampling methods (e.g. visual record, Malaise trap, light trap) may still increase the species records for Bodoquena Plateau, highlighting the importance of this region, which lies into two highly threatened biomes.

Our new records extend the register of distribution to many species, information considered critical for the definition of their threatened status (IUCN 2016). When considering the IUCN red list, the Odonata species of the Neotropical region have a high number of species listed as Data Deficient (Clausnitzer et al. 2009) or without status definition. In this study, $2 / 3$ of the species do not have classification, which reinforces this information gap related to conservation for the group. Regarding the species classified as threatened, Elga newtonsantosi and Micrathyria pseudhypodidyma have limited information available about their distribution (number of locations), population size, and ability to survive in reduced habitat (von Ellenrieder

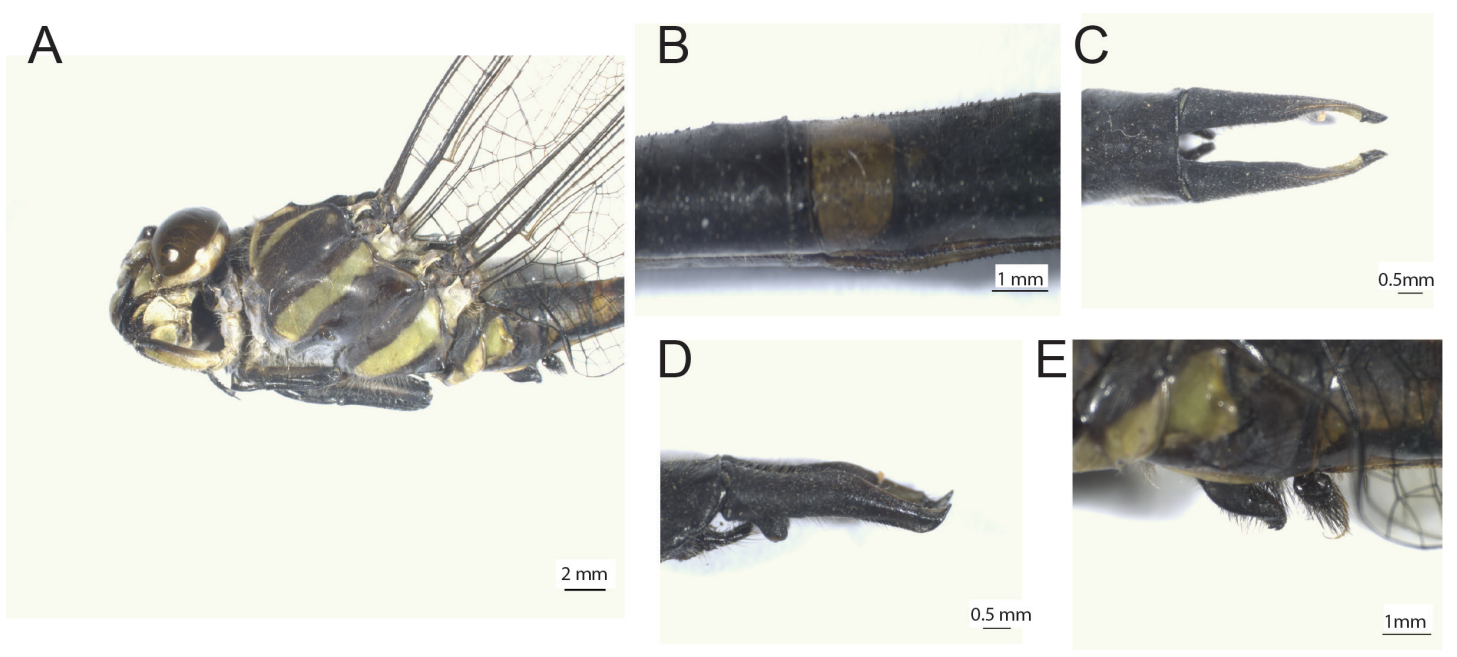

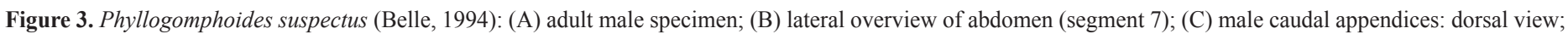
(D) male caudal appendices: lateral view; (E) male genital hamule: lateral view. 
2009b, von Ellenrieder 2009c). From a conservation perspective, the knowledge about threatened species distributions is crucial for biodiversity management, especially for the development and implementation of conservancy plans. Thus, both of them shall be targets of governmental protection plan, as required in the Brazilian legislation on endangered species. In summary, this study highlighted the Bodoquena Plateau as an important habitat for odonates, including species classified as threatened.

\section{Acknowledgements}

We are grateful to several colleagues for their help: Janira Martins Costa, Cesar Carriço, Danielle Anjos-Santos, National Museum (MNRJ, Rio de Janeiro, Brazil), Mr. Javier Muzon, Alexandro Del Placio, Federico Lozano and Lia Ramos, National University of Avellaneda (Avellaneda, Argentina) helped us with the identifications. We also thank the projects Long Term Ecological Research in the Bodoquena Plateau (LTER-Serra da Bodoquena), NGO Neotropica do Brasil, Observagua Project and the hotel Cabanas staff. RK receives a scholarship of CAPES and grants from Rufford Foundation (Dragonflies of Serra da Bodoquena Project, RSGF 19689-1). FOR received a productivity grant, no. 09908/2013-2, from CNPq. This study was partially funded by the Fundect.

\section{Authors' Contributions}

KOROIVA, R., RODRIGUES, M., VALENTE-NETO: contribution to data collection.

KOROIVA, R., RODRIGUES, M., VALENTE-NETO, F. and ROQUE, F.O: contribution to data analysis and interpretation.

KOROIVA, R., RODRIGUES, M., VALENTE-NETO, F. and ROQUE, F.O: contribution to manuscript preparation.

KOROIVA, R., RODRIGUES, M., VALENTE-NETO, F. and ROQUE, F.O: contribution to critical revision, adding intelectual content.

\section{Conflicts of interest}

The authors declare that they have no conflict of interest related to the publication of this manuscript.

\section{References}

ABBOTT, J.C. 2015. Dragonflies of Texas: A field guide. University of Texas Press, Austin.

ANJOS-SANTOS, D. \& COSTA, J.M. 2006. A revised checklist of Odonata (Insecta) from Marambaia, Rio de Janeiro, Brazil with eight new records. Zootaxa 1300: 37-50.

BELLE, J. 1994. On five species of Phyllogomphoides Belle, 1970, from Brazil with the descriptions of three new taxa (Odonata: Gomphidae). Zool. meded. 68: 73-85.

BRASIL. 2016. Áreas prioritárias para conservação, uso sustentável e repartição dos benefícios da biodiversidade. Ministério do Meio ambiente. World Wide Web publication. http://www.mma.gov.br/images/arquivo/80049/Areas\%20 Prioritarias/Cerrado\%20e\%20Pantanal/FICHAS\%20Areas\%20Prioritarias\%20 Cerrado\%20e\%20Pantanal 2\%20atualizacao\%2022ago16.pdf (last access at 23/November/2016).

CLAUSNITZER, V., KALKMAN, V.J., RAM, M., COLLEN, B., BAILLIE, J.E.M., BEDJANIC, M., DARWALL, W.R.T., DIJKSTRA, K.B., DOW, R., HAWKING, J., KARUBE, H., MALIKOVA, E., PAULSON, D., SCHÜTTE, K., SUHLING, F., VILLANUEVAM, R.J., VON ELLENRIEDER, N. \& WILSON, K. 2009. Odonata enter the biodiversity crisis debate: the first global assessment of an insect group. Biol. Conserv. 142: 1864-1869.

COLWELL, R.K. 2013. EstimateS 9.1.0. World Wide Web publication. http://viceroy. eeb.uconn.edu/estimates (last access at 23/November/2016).
COSTA, J.M., MACHADO, A.B.M., LENCIONI, F.A.A. \& SANTOS, T.C. 2000. Diversidade e distribuição dos Odonata (Insecta) no estado de São Paulo, Brasil. Pub. Avul. Mus. Nac. 80(1): 1-27.

COSTA, J.M. \& OLDRINI, B.B. 2005. Diversidade e distribuição dos Odonata (Insecta) no Estado do Espírito Santo, Brasil. Publ. Avulsas Mus. Nac. 107:1-15.

DALZOCHIO, M.S., COSTA, J.M. \& UCHÔA, M.A. 2011a. Diversity of Odonata (Insecta) in lotic systems from Serra da Bodoquena, Mato Grosso do Sul State, Brazil. Rev. Bras. Entomol. 5(1): 88-94.

DALZOCHIO, M.S., SOUZA, L.O.I., UCHÔA, M.A. \& COSTA, J.M. 2011b. First records of Odonata (insecta) From the Bodoquena Mountains, Mato Grosso do Sul, Brazil. EntomoBrasilis 4(3): 135-138.

DINIZ-FILHO, J.A.F., DE-MARCO, P. \& HAWKINS, B.A. 2010. Defying the curse of ignorance: perspectives in insect macroecology and conservation biogeography. Insect Conserv. Diver. 3: 172-179.

GARRISON, R. \& VON ELLENRIEDER, N. 2016. A synonymic list of the New World Odonata. World Wide Web publication. http://www.odonatacentral.org/ docs/NWOL.pdf (last access at 23/November/2016).

GARRISON, R.W., VON ELLENRIEDER, N. \& LOUTON, J.A. 2006. Dragonfly genera of the new world: an illustrated and annotated key to the Anisoptera. The John Hopkins University Press, Baltimore.

GARRISON, R.W., VON ELLENRIEDER, N. \& LOUTON, J.A. 2010. Damselfly genera of the new world: an illustrated and annotated key to the Zygoptera. The John Hopkins University Press, Baltimore.

GOTELLI, N.J. \& COLWELL, R.K. 2001. Quantifying biodiversity: procedures and pitfalls in the measurement and comparison of species richness. Ecol. Lett. 4: 379-391.

HECKMAN, C.W. 2006. Encyclopedia of South American aquatic insects: Odonata - Anisoptera. Springer, Dordrecht.

HECKMAN, C.W. 2010. Encyclopedia of South American aquatic insects: Odonata - Zygoptera. Springer, Dordrecht.

IUCN (International Union for Conservation of Nature). 2016. The IUCN Red List of Threatened Species, Version 2016-2. World Wide Web publication. http:// www.iucnredlist.org. (last access at 23/November/2016).

KALKMAN, V.J., CLAUSNITZER, V., DIJKSTRA, K.D.B., ORR, A.G., PAULSON, D.R. \& VAN TOL, J. 2008. Global diversity of dragonflies (Odonata) in freshwater. Hydrobiologia 595: 351-363.

KLEIN, F.M., ESCANDOLHERO, J.P.O., LUCCHESE, N.R., MERCANTE, M.A., FÁVERO, S. \& RODRIGUES, S.C. 2011. Educação ambiental e o ecoturismo na Serra da Bodoquena em Mato Grosso do Sul. Soc. Nat. 23(2): 311-321.

KOROIVA, R., VALENTE-NETO, F., RODRIGUES, M.E. \& ROQUE, F.O. 2016. As reveladoras libélulas da Bodoquena. Ciência Pantanal 2: 26-27.

LENCIONI, F.A.A. 2005. Damselflies of Brazil: An Illustrated Identification Guide - 1 - Non-Coenagrionidae Families. All Print Editora, São Paulo.

LENCIONI, F.A.A. 2006. The Damselflies of Brazil: An Illustrated Identification Guide 2 - Coenagrionidae. All Print Editora, São Paulo.

MELO, A.S. \& FROELICH, C.G. 2001. Evaluation of methods for estimating macroinvertebrate species richness using individual stones in tropical streams. - Freshwat. Biol. 46: 711-721.

NÓBREGA, C.C. \& DE MARCO, P. jr. 2011. Unprotecting the rare species: a niche-based gap analysis for odonates in a core Cerrado área. Divers. Distrib. 17(3): 491-505.

PCBAP (Plano de Conservação da Bacia do Alto Paraguai - Pantanal). 1997. Análise integrada e prognóstica da Bacia do Alto Paraguai. MMA/SEMAM/ PNMA, Brasília.

RENNER, S., PERICO, E, \& SAHLEN, G. 2016. List of Odonates from the Floresta Nacional de São Francisco de Paula (FLONA - SFP), with two new distribution records for Rio Grande do Sul, Brazil. Biota Neotrop. 16(3): e20150132. http:// www.biotaneotropica.org.br/v16n3/pt/download?inventory+bn00616032016+ item. (last access at 23/November/2016).

RODRIGUES, M.E. 2016. Odonates as indicators of landscape change in a region of the Cerrado. 2016. Tese de doutorado, Universidade Federal de Mato Grosso do Sul, Campo Grande. 
RODRIGUES, M.E., KOROIVA, R., RAGALZI-DA-SILVA, E. \& MOURA, E.B. 2016a. Mecistogaster linearis (Fabricius) (Odonata: Coenagrionidae): First Record from Mato Grosso do Sul State, Brazil. EntomoBrasilis. 9: 212-215.

RODRIGUES, M.E., ROQUE, F.O., OCHOA-QUINTERO, J.M., PENA, J.C.C., SOUSA, D.C. \& DE-MARCO, P. Jr. 2016b. Nonlinear responses in damselfly community along a gradient of habitat loss in a savanna landscape. Biol. Conserv. 194: 113-120.

RODRIGUES, M.E. \& ROQUE, F.O. 2017. Checklist de Odonata do estado de Mato Grosso do Sul, Brasil. Iheringia Ser. Zool. 107(suppl.): e2017117.

ROQUE, F.O., OCHOA-QUINTERO, J.M., RIBEIRO, D.B., SUGAI, L.S.M, COSTA-PEREIRA, R., LOURIVAL, R. \& BINO, G. 2016. Upland habitat loss as a threat to Pantanal wetlands. Conserv. Biol. 30 (5): 1131-1134.

SOUZA, L.O.I. 2003. A Influência dos Fatores Ambientais na Distribuição da Fauna de Odonata (Insecta) em Riachos da Serra da Bodoquena, MS. Dissertação de Mestrado, Universidade Federal de Mato Grosso do Sul, Campo Grande.

SOUZA, M.M., SOUZA, B., PEREIRA, M.C.S.A. \& MACHADO, A.B.M. 2013. List of Odonates from Mata do Baú, Barroso, Minas Gerais, Brazil. Check List 9: 1367-1370.

VALENTE-NETO, F. 2015. Ecological drivers of aquatic metacommunities in a riverine network. 2015. Tese de doutorado, Universidade Federal de Mato Grosso do Sul, Campo Grande.
VALENTE-NETO, F., ROQUE, F.O., RODRIGUES, M.E., JUEN, L. \& SWAN, C.M. 2016. Toward a practical use of Neotropical odonates as bioindicators: Testing congruence across taxonomic resolution and life stages. Ecol. Indic. 61: 952-959.

VIANNA, D.M. \& DE-MARCO, P. Jr. 2012. Higher-taxon and cross-taxon surrogates for odonate biodiversity in Brazil. Nat. Conservacao 10: 34-39.

VON ELLENRIEDER, N. 2009a. Phyllogomphoides suspectus. The IUCN Red List of Threatened Species. World Wide Web publication. http://dx.doi. org/10.2305/IUCN.UK.2009-2.RLTS.T159007A5305086.en (last access at 23/November/2016).

VON ELLENRIEDER, N. 2009b. Elga newtonsantosi. The IUCN Red List of Threatened Species. World Wide Web publication. http://dx.doi.org/10.2305/ IUCN.UK.2009-2.RLTS.T159064A5318685.en (last access at 23/November/2016).

VON ELLENRIEDER, N. 2009c. Micrathyria pseudhypodidyma. The IUCN Red List of Threatened Species. World Wide Web publication. http://dx.doi. org/10.2305/IUCN.UK.2009-2.RLTS.T159112A5315873.en (last access at 23/November/2016).

Received: 05/12/2016

Revised: 02/06/2017

Accepted: 10/07/2017

Published online: 31/07/2017 